\title{
Random Matrix Approach to Quantum Adiabatic Evolution Algorithms
}

\author{
A. Boulatov and V. N. Smelyanskiy
}

May 7, 2004

\begin{abstract}
We analyze the power of quantum adiabatic evolution algorithms (QAEA) for solving random NP-hard optimization problems within a theoretical framework based on the random matrix theory (RMT). We present two types of the driven RMT models. In the first model, the driving Hamiltonian is represented by Brownian motion in the matrix space. We use the Brownian motion model to obtain a description of multiple avoided crossing phenomena. We show that the failure mechanism of the QAA is due to the interaction of the ground state with the "cloud" formed by all the excited states, confirming that in the driven RMT models, the Landau-Zener mechanism of dissipation is not important. We show that the QAEA has a finite probability of success in a certain range of parameters, implying the polynomial complexity of the algorithm. The second model corresponds to the standard QAEA with the problem Hamiltonian taken from the Gaussian Unitary RMT ensemble (GUE). We show that the level dynamics in this model can be mapped onto the dynamics in the Brownian motion model. However, the driven RMT model always leads to the exponential complexity of the algorithm due to the presence of the long-range intertemporal correlations of the eigenvalues. Our results indicate that the weakness of effective transitions is the leading effect that can make the Markovian type QAEA successful.
\end{abstract}




\section{Introduction}

The quantum adiabatic algorithms (QAA) are designed for solving combinatorial search and optimization problems based on the quantum evolution of the relevant systems [1]. These algorithms are based on adiabatic theory of Quantum Mechanics, stating that the quantum state is closely following an instantaneous ground state of a slow-varying in time control Hamiltonian, provided that the initial state was chosen to be a ground state of the initial Hamiltonian. At the initial moment of time the control Hamiltonian has a simple form with the known ground state that is easy to prepare and at the final moment of time it coincides with the "problem" Hamiltonian $H_{P}$ which ground state encodes the solution of classical optimization problem in question. It also can be chosen to reflect the bit-structure and cost spectrum of the problem. For example,

$$
\begin{aligned}
& H_{P}=\sum_{\mathbf{z}} E_{\mathbf{z}}|\mathbf{z}\rangle\langle\mathbf{z}| \\
& |\mathbf{z}\rangle=\left|z_{1}\right\rangle_{1} \otimes\left|z_{2}\right\rangle_{2} \otimes \cdots \otimes\left|z_{n}\right\rangle_{n} .
\end{aligned}
$$

Here $E_{\mathbf{z}}$ is a cost function defined on a set of $2^{n}$ binary strings $\mathbf{z}=\left\{z_{1}, \ldots, z_{n}\right\}$ $z_{j}=0,1$, each containing $n$ bits. The summation in (1) is over $2^{n}$ states $|\mathbf{z}\rangle$ forming the computational basis of a quantum computer with $n$ qubits. State $\left|z_{j}\right\rangle_{j}$ of the $j$-th qubit is an eigenstate of the Pauli matrix $\hat{\sigma}_{z}$ with eigenvalue $1-2 z_{j} \pm 1$ ). If at the end of QAA the quantum state is sufficiently close to the ground state of $H_{P}$ then the solution to the optimization problem can be retrieved by the measurement.

It was shown $[2,32]$ that the system can be trapped during the QAA in the local minimum of a cost function for a time that grows exponentially in the problem size $n$. It was also shown [2] that an exponential delay time in quantum adiabatic algorithm can be interpreted as a quantum-mechanical tunneling of an auxiliary large spin system.

The paper [4] demonstrates that tunneling can be avoided by a suitable modification of the QAA evolution "paths" with the same initial and final wave functions. The authors presented a general approach using the "stochastic" paths and numerical results indicated that the HWP may be solved in polynomial time with finite probability. This approach was further developed in [5], where the complete analytic characterization of the "stochastic" path evolution and probability of success was obtained. 
In present paper, we analyze the performance of the Quantum Adiabatic Algorithm (QAA) in the framework of the Random Matrix Theory (RMT). We show that the runtime of the algorithm should be typically exponentially large in the number of qubits $n$.in order to have a polynomial probability of success for the quantum evolution algorithm. This implies that the QAA typically has exponential complexity.

\section{Quantum Adiabatic Evolution Algorithm}

In a standard QAA $[1]$, one specifies the time-dependent Hamiltonian $H(t)$

$$
H(t)=\alpha(t) H_{D}+\beta(t) H_{P}
$$

with the initial and terminal conditions

$$
\begin{aligned}
\alpha(0)=1, & & \beta(0)=0, \\
\alpha(T)=0, & & \beta(T)=1 .
\end{aligned}
$$

The Hamiltonian (2) guides the quantum evolution of the state vector $|\psi(t)\rangle$ from $t=0$ to $t=T$, the run time of the algorithm, and $H_{P}$ is the "problem" Hamiltonian given in (1). In case when the parametric evolution of the Hamiltonian (2) is sufficiently slow, the Adiabatic theorem assures that the system initially occupving the ground state of the driver $H_{D}=H(0)$, parametrically evolves into the ground state of $H_{P}=H(T)$. The standard estimate of the run time that provides such adiabatic evolution, is well known and given by [1]

$$
T \gtrsim 1 / \Delta^{2}
$$

where $\Delta$ is the lowest value of the energy gap between the ground and the first excited adiabatic states.

The "driver" Hamiltonian $H_{D}$ is designed to cause the transitions between the eigenstates of $H_{P}$. According to the above discussion, no prepares the initial state of the system $|\psi(0)\rangle$ to be a ground state of $H(0)=H_{D}$. It is typically constructed assuming no knowledge of the solution of the classical optimization problem and related ground state of $H_{P}$. In the simplest case

$$
H_{D}=-C \sum_{j=0}^{n-1} \sigma_{x}^{j}, \quad|\psi(0)\rangle=2^{-n / 2} \sum_{\mathbf{z}}|\mathbf{z}\rangle
$$


where $\sigma_{x}^{j}$ is a Pauli matrix for $j$-th qubit and $C>0$ is some scaling constant. Consider instantaneous eigenstates $\left|\phi_{k}(\tau)\right\rangle$ of $H(\tau)$ with energies $E_{k}(\tau)$ arranged in non-decreasing order at any value of $\tau \in(0,1)$

$$
H\left|\phi_{k}\right\rangle=E_{k}\left|\phi_{k}\right\rangle, \quad k=0,1, \ldots, 2^{n}-1 .
$$

Expanding the non-stationary wave function of the system in terms of the instantaneous adiabatic basis (which is complete for any fixed $\tau \in(0,1)$ ), we obtain $([6])$ :

$$
|\psi(t)\rangle=\sum_{k=0}^{N} C_{k}(t) \exp \left[-i \int^{t} d t^{\prime} E_{k}\left(t^{\prime}\right)\right]\left|\phi_{k}(t)\right\rangle,
$$

with $N=2^{n}-1$ complex coefficients $\left\{C_{k}(t)\right\}$ satisfying the normalization condition $\sum_{k=0}^{N}\left|C_{k}(t)\right|^{2}=1$ for $t \in(0, T)$, and we use the units with $\hbar=1$. Note that each quantity $\left|C_{k}(t)\right|^{2}(k=0, \ldots N)$ is the probability to find the system in the instantaneous adiabatic eigenstate $\left|\phi_{k}(t)\right\rangle$ at time $t$. According to the above discussion and taking into account the normalization condition, the initial conditions for (7) are given by

$$
C_{0}(0)=1, \quad C_{k}(0)=0, \quad k=1, \ldots, N .
$$

Under the assumption that the populations of the excited states of the instantaneous Hamiltonian are small, the Schroedinger equation applied to (7) yields $[6]$

$$
\frac{\partial}{\partial t} C_{k}(t)=\left\langle\phi_{k}(t)\left|\frac{\partial}{\partial t}\right| \phi_{0}(t)\right\rangle \exp \left\{-i \int^{t} d t^{\prime}\left[E_{k}\left(t^{\prime}\right)-E_{0}\left(t^{\prime}\right)\right\}\right\}
$$

describing the dynamics of the non-adiabatic transitions from the ground state $k=0$ to the states with $k=1, \ldots, N$. Taking into account (9), we obtain the probability $P_{0}(t)$ for the system to remain in adiabatic ground state in the form

$$
P_{0}(t)=1-\Gamma(t),
$$

with $\Gamma(t)$ being a total probability of escape from the ground state, or failure of the QAA, defined by 


$$
\Gamma(t)=\sum_{k=1}^{N} \int_{0}^{t} d t^{\prime} \int_{0}^{t} d t^{\prime \prime}\left(\frac{\partial}{\partial t^{\prime}}\right)_{k 0}\left(\frac{\partial}{\partial t^{\prime \prime}}\right)_{0 k} \exp \left\{-i \int_{t^{\prime}}^{t^{\prime \prime}} d \tau \omega_{k 0}(\tau)\right\}
$$

In (11), $\left(\frac{\partial}{\partial t}\right)_{k 0}=\left\langle\phi_{k}(t)\left|\frac{\partial}{\partial t}\right| \phi_{0}(t)\right\rangle$ is the matrix element of the operator $\frac{\partial}{\partial t}$ and $\omega_{k 0}(t)=E_{k}(t)-E_{0}(t)$ is the transition frequency between the ground and the $k$-th excited adiabatic state. In what follows, we will analyze the probability (11) for a particular case of a driven RMT model for the problem Hamiltonian.

\section{Random Matrix Analysis of QAA}

According to the above discussion, the problem Hamiltonian of QAA should have a complex ground state encoding the optimal solution of the initial optimization problem. As a simple "limiting case" of such a complex Hamiltonian, one may suggest a random Hamiltonian sampled from one of the symmetry ensembles of the Random Matrix Theory (RMT) [7]. It has been shown that the "random" Hamiltonians belonging to a certain symmetry class (say Unitary Ensemble, or GUE), give a good description of the complex physical systems, which do not possess any specific conservation laws and are only characterized by the symmetry class with respect to the change of basis (unitary transformations) [7], [8]. We will give more motivations for such choice in a subsequent section. In this section, we will analyze a specific case of such a "random" problem Hamiltonian,. when the matrix elements of the problem Hamiltonian evolve according to the Ornstein-Uhlenbeck processes [18]

$$
\frac{d H_{k j}(t)}{d t}=-\gamma H_{k j}(t)+\sigma \xi_{k j}
$$

where $\gamma>0$ describes the dissipation, $\sigma>0$ the noise amplitude and $z_{k j}(t)$ correspond to $N^{2}$ independent Langevin sources

$$
\left\langle\xi_{k j}(t), \xi_{k^{\prime} j^{\prime}}\left(t^{\prime}\right)\right\rangle=\delta_{k k^{\prime}} \delta_{j j^{\prime}} \delta\left(t-t^{\prime}\right)
$$

In (13), $\tilde{\delta}_{k l}$ stands for the Kronecker symbol and $\delta\left(t-t^{\prime}\right)$ is a Dirac's delta function. The formal solution of the stochastic differential equation (12) is given by 


$$
H_{k j}(t)=H_{k j}(0) \exp [-\gamma t]+\sigma \int_{0}^{t} d t^{\prime} \xi_{k j}\left(t^{\prime}\right) \exp \left[-\gamma\left(t-t^{\prime}\right)\right]
$$

where $H_{k j}(0)$ is an arbitrary Hermitian matrix representing the "initial condition" for the driven random matrix $H_{k j}(t=0)=H_{k j}(0)$.

\subsection{Angular Decomposition in GUE}

The "angular coordinates" for the Hermitian matrices $H$ belonging to the General Unitary Ensemble (GUE) [7] are defined by

$$
H=U \Lambda U^{+},
$$

where the diagonal matrix $\Lambda_{i j}=\delta_{i j} \lambda_{i}$ represents the eigenvalues $\left\{\lambda_{i}\right\}$ of $H$ which are invariant with respect to the unitary transformations $U_{i j}=\psi_{j}^{(i)}$ characterizing the $j$-th component of the $i$-th eigenvector belonging to the eigenvalue number $i$. Therefore, the unitary matrix $U$ represents a particular choice of the basis and the vector $\Lambda$ is basis-invariant. Note that the unitarity conditions $U^{+} U=U U^{+}=I$ where $I$ is a unit matrix, correspond to the orthonormality and completeness of the basis $\psi^{(i)}$. Differentiating (15) and taking into account the unitarity conditions, we obtain

$$
U^{+} d H U=d \Lambda+[d \Omega, d \Lambda]
$$

where the generalized "angular" motion is defined by

$$
d \Omega=U^{+} d U,
$$

and $[\ldots]$ is a commutator, i.e. $[d \Omega, d \Lambda]=d \Omega d \Lambda-d \Lambda d \Omega$. Making use of (16), we obtain the equations of motion for the eigenvalues and angles $\Omega$ in the "local" basis with $U=I$, in the form

$$
\begin{aligned}
\frac{d \lambda_{k}}{d t} & =\sigma \xi_{k k}-\gamma \lambda_{k}+\left(\sum_{l \neq k} \frac{\sigma^{2}}{\lambda_{k}-\lambda_{l}}\right), \\
\frac{d \Omega_{k j}}{d t} & =\frac{\sigma}{\lambda_{j}-\lambda_{k}} \xi_{k j} .
\end{aligned}
$$


From (15), it follows that

$$
\begin{aligned}
\frac{d \Omega_{k 0}}{d t} & =\left\langle\phi_{k}(t)\left|\frac{\partial}{\partial t}\right| \phi_{0}(t)\right\rangle=\left(\frac{\partial}{\partial t}\right)_{k 0}, \\
\lambda_{k}(t)-\lambda_{0}(t) & =E_{k}(t)-E_{0}(t)=\omega_{k 0}(t),
\end{aligned}
$$

establishing the connection between the probability (11) and the angular decomposition variables.

\subsection{Optimal Trajectories for Driven RMT}

The first of equation (18) describes the many-particle system in $1 D$ undergoing the Brownian motion and interacting with the long-range pairwise potential $\sim 1 / r[7],[8]$. Importantly, the dynamics of the eigenvalues $\Lambda(t)$ does not depend on the eigenvector dynamics $\Omega(t)$. Since the velocities of the Brownian particles contain the noise terms, each realization of the general many-particle trajectory in the phase space is drawn from the random distribution characterized by a particular choice of the Langevin source trajectories $\xi_{k j}(t)$. Therefore, the probability of success of the QAA (11) becomes a functional defined on each of the Langevin source trajectories. Since these trajertories are random, this probability $P_{\mathrm{n}}$ is characterized by the distribution in the general phase space of the problem, with the "distribution density functional" defined on the realizations of the Langevin sources and therefore on the phase coordinates of the system, in the form [23]

$$
W[\Lambda(t), \Omega(t)]=\exp \{-S[\Lambda(t), \Omega(t)]\},
$$

with the action functional $S$ denfned through the LagIangian function

$$
S=\int_{0}^{T} d t^{\prime} L\left[\Lambda\left(t^{\prime}\right), \dot{\Lambda}\left(t^{\prime}\right), \Omega\left(t^{\prime}\right), \dot{\Omega}\left(t^{\prime}\right)\right],
$$

where $\dot{\Lambda}=d \Lambda / d t$ and $\dot{\Omega}=d \Omega / d t$, and $L$ is the Lagrangian. Following the standard procedure, the Lagrangian for the system (18) is given by [22] 


$$
\begin{aligned}
L & =\frac{1}{2 \sigma^{2}}\left\{\sum_{k}\left[\left(\dot{\lambda_{k}}-v_{k}\right)^{2}+\sigma^{2} v_{k}^{\prime}\right]+\sum_{k, j}\left(\lambda_{k}-\lambda_{j}\right)^{2}\left(\dot{\Omega}_{k j}\right)\right\}^{2}, \\
v_{k} & =-\gamma \lambda_{k}+\left(\sum_{l \neq k} \frac{\sigma^{2}}{\lambda_{k}-\lambda_{l}}\right),
\end{aligned}
$$

with $v_{k}^{\prime}(\Lambda)=\partial v_{k}(\Lambda) / \partial \lambda_{k}$. Making use of (11), (18), and (19), we obtain the expected probability of failure $\langle\Gamma(t)\rangle=1-\left\langle P_{0}(t)\right\rangle$ in the form

$$
\langle\Gamma(t)\rangle=\frac{\iint D \Lambda D \Omega W[\Lambda, \Omega] \sum_{k=1}^{N} \int_{0}^{t} d t^{\prime} \int_{0}^{t} d t^{\prime \prime} \dot{\Omega}_{k 0}\left(t^{\prime}\right) \dot{\Omega}_{0 k}\left(t^{\prime \prime}\right) \exp \left\{-i \Phi_{k 0}\right\}}{\iint D \Lambda D \Omega W[\Lambda, \Omega]}
$$

where $\Phi_{k 0}\left(t^{\prime}, t^{\prime \prime}\right)=\int_{t^{\prime}}^{\iota^{\prime \prime}} d \tau \omega_{k 0}(\tau), \int D \Lambda \int D \Omega W[\Lambda, \Omega]$ denote the functional integration over all trajectories with the weight defined as a functional probability density (20), and the denominator is included into (23) for normalization. From (22), it follows that the "angular" part enters as an additive quadratic term into the Lagrangian, and therefore the integration over the angular variables $\int D \Omega$ in (23) can be done explicitly [23], with the result

$$
\frac{\int D \Omega W[\Lambda, \Omega] \dot{\Omega}_{k 0}(t) \dot{\Omega}_{0 k}\left(t^{\prime}\right)}{\iint D \Lambda D \Omega W[\Lambda, \Omega]}=\left(\frac{\sigma^{2}}{\left[\lambda_{k}(t)-\lambda_{0}(t)\right]^{2}}\right) \delta\left(t-t^{\prime}\right) .
$$

Combining (23) and (24), we obtain

$$
\langle\Gamma(t)\rangle=\sum_{k=1}^{N} \int D \Lambda \frac{\exp \left(-S_{e f f}[\Lambda]\right)}{\int D \Lambda^{\prime} \exp \left(-S_{e f f}\left[\Lambda^{\prime}\right]\right)} \int_{0}^{t} d t^{\prime} \frac{\sigma^{2}}{\left[\lambda_{k}\left(t^{\prime}\right)-\lambda_{0}\left(t^{\prime}\right)\right]^{2}},
$$

where the effective action $S_{\text {eff }}[\Lambda]$ is taken with the "truncated" Lagrangian $L_{e f f}$

$$
L_{e f f}=\frac{1}{2 \sigma^{2}} \sum_{k}\left[\left(\dot{\lambda_{k}}-v_{k}(\Lambda)\right)^{2}+\sigma^{2} v_{k}^{\prime}(\Lambda)\right]
$$


The evaluation of (25) is still very difficult, since it involves the averaging over the unknown level distribution.

For the many-body system with exponential number of particles $N=2^{n}$, the distribution of the system's trajectories in the phase space is expected to be "sharp" around the optimal trajectory, defined from the variation principle [21]. The variational principle takes the form of minimum action

$$
S_{\min }=\min _{\{\Lambda(t), \Omega(t)\}}\{S[\Lambda(t), \Omega(t)]\},
$$

Given (22), the optimal trajectories are obtained from the minimum action principle (27) as the solutions of the Euler-Lagrange equations

$$
\frac{d}{d t}\left(\frac{\partial L}{\partial \dot{q}_{i}}\right)=\left(\frac{\partial L}{\partial q_{i}}\right)
$$

where $q_{i}=\left\{\lambda_{i}\right\}$ represent the generalized coordinates [9]. Combining (22) and $(28)$, we obtain

$$
\ddot{\lambda_{k}}(t)-\sum_{j} \dot{\lambda}_{j}\left(\frac{\partial v_{k}}{\partial \lambda_{j}}-\frac{\partial v_{j}}{\partial \lambda_{k}}\right)=-\frac{\partial}{\partial \lambda_{k}} U_{e f f}(\Lambda),
$$

with the effective potential given by

$$
U_{e f f}(\Lambda)=-\frac{1}{2} \sum_{k}\left(v_{k}^{2}(\Lambda)+\sigma^{2} \frac{\partial \eta_{k}(\Lambda)}{\partial \lambda_{k}}\right) .
$$

Combining (22), (29) and (30) yields

$$
\begin{aligned}
\ddot{\lambda_{k}}(t) & =-\frac{\partial}{\partial \lambda_{k}} U_{e f f}(\Lambda), \\
U_{e f f}(\Lambda) & =-\frac{\gamma^{2}}{2} \sum_{k} \lambda_{k}^{2} .
\end{aligned}
$$

The details of the calculations leading to (31) are presented in Appendix A. The effective potential in (31) can also be derived from the Fokker-Plank equation describing the time evolution of the $N$-particle level distribution function [18]. In general, that procedure leads to the dynamic equations 
describing the many-particle system in $1 D$ with pairwise interactions. In a particular case of the unitary ensemble, the pairwise interaction vanishes and the problem reduces to the $N$-particle non-interacting system. Therefore, we finally have the following description of the optimal trajectories

$$
\ddot{\lambda_{k}}(t)-\gamma^{2} \lambda_{k}=0
$$

The equations (32) are of the second order and therefore require two initial (or boundary) conditions for each $k=0, \ldots N$. Since (18) are the first order stochastic differential equations, we only have one initial condition for the optimal trajectories

$$
\lambda_{k}(t=0)=\lambda_{k}(0),
$$

for each $k$ and the final points $\lambda_{k}(t=T)$ are not fixed. We impose the additional boundary conditions at $t=T$ in the form

$$
-\gamma \lambda_{k}(T)+\left(\sum_{l \neq k} \frac{\sigma^{2}}{\lambda_{k}(T)-\lambda_{l}(T)}\right)=0
$$

which insure that at $t=T$ the system belongs to the equilibrium unitary ensemble. Specifically, (34) corresponds to the standard GUE distribution [7], [8]. As we show in Appendix B, the boundary conditions (34) can also be obtained in the long-time limit for the optimal trajectories in case when the terminal conditions at $t=T$ are not fixed and have to be self-consistently derived from the optimality property of the trajectories. In fact, we show that the terminal conditions for the "arbitrary" optimal trajectories are exponentially close to the equilibrium described by (34). This implies that the "spread" of the optimal trajectories is exponentially small in the large-time limit, confirming the validity of the approach described above.

The equations (32) with (33) and (34), provide a close set characterizing the optimal trajectories of the initial many-body system. In the subsequent section, the probability of success of the QAA will be evaluated using the solutions for the optimal trajectories.

\subsection{Probability of Success for the QAA}

As we have mentioned above, for the system with exponential number of particles $N$, the distribution of trajectories in the phase space is expected 
to be "sharp" around the optimal trajectory. Following this argument, we observe that with exponential accuracy [21]

$$
\langle\Gamma(t)\rangle=\sum_{k=1}^{N} \int_{0}^{t} d t^{\prime} \frac{\sigma^{2}}{\left[\lambda_{k}^{*}\left(t^{\prime}\right)-\lambda_{0}^{*}\left(t^{\prime}\right)\right]^{2}},
$$

where $\left\{\lambda_{k}^{*}\right\}$ correspond to the optimal trajectories. In order to simplify the notations, we will denote $\left\{\lambda_{\dot{k}}^{*}\right\}=\left\{\lambda_{k}\right\}$, i.e. drop the superscript. This will not cause the confusion, since we will only consider the optimal trajectories in the rest of the paper. From (32) with (33) and (34), we obtain

$$
\lambda_{k}(t)=\lambda_{k}(0) \frac{\sinh [\gamma(T-t)]}{\sinh (\gamma T)}+\lambda_{k}(T) \frac{\sinh (\gamma t)}{\sinh (\gamma T)} .
$$

The final "coordinates" $\left\{\lambda_{k}(T)\right\}$ are defined from (34) and correspond to the standard equilibrium GUE distribution [7], [8] at $t=T$. Substituting (36) into (35), we obtain

$$
\langle\Gamma(T)\rangle=\sum_{k=1}^{N} \int_{0}^{T} d t^{\prime} \frac{\sigma^{2} \sinh ^{2}(\gamma T)}{\left[\omega_{k 0}(0) \sinh \left[\gamma\left(T-t^{\prime}\right)\right]+\omega_{k 0}(T) \sinh \left(\gamma t^{\prime}\right)\right]^{2}} .
$$

The integration in (37) can be done explicitly, with the result

$$
\langle\Gamma(T)\rangle=\frac{\sigma^{2}}{\gamma} \sinh (\gamma T) \sum_{k=1}^{N} \frac{1}{\omega_{k 0}(0) \omega_{k 0}(T)} .
$$

Making use of the terminal condition (34), the probability (38) can be estimated as

$$
\langle\Gamma(T)\rangle=\sinh (\gamma T) \lambda_{0}(T)\left\langle\frac{1}{\omega_{k 0}(0)}\right\rangle .
$$

Since the Hamiltonian at $t=0$ is given by (5), the average inverse frequency in (39) is bounded

$$
\frac{1}{n} \leq\left\langle\frac{1}{\omega_{k 0}(0)}\right\rangle \leq 1
$$

On the other hand, according to (34), we have 


$$
\lambda_{0}(T)=\frac{\sigma \sqrt{2 N}}{\sqrt{\gamma}} .
$$

Since the spectrum of the problem Hamiltonian is bounded in the largen limit, the ground state energy $\lambda_{0}(T)$ should not depend on $n$. This is satisfied if

$$
\sigma_{0}^{2}=\frac{\sigma^{2}}{\gamma} \sim \frac{1}{N}
$$

which is a common scaling in RMT models [7]. As we will see in the subsequent section, the scaling (42) naturally occurs in the QAEA framework. In Appendix $C$, we show that the equilibrium $N$-level distribution only depends on the parameter $\sigma_{0}^{2}=\sigma^{2} / \gamma$, confirming that the condition (42) is sufficient for the spectrum to be bounded in a finite interval.

Given the estimate (40), the summation over the energy spectrum is performed using the exact equilibrium condition (34), and therefore the result (39) is exact. This means that (39) is based on the exact summation over the discrete spectrum valid for any total number of states $N$ and does not refer to any continuous level distribution arising in the large $N$ limit. In the large $N$ limit, the equilibrium density of states (DOS) for the GUE ensemble is given by the famous "semicircle" law $[7],[8]$

$$
\rho(\lambda)=\frac{2 N}{\pi \lambda_{0}^{2}} \sqrt{\lambda_{0}^{2}-\lambda^{2}}, \quad-\lambda_{0} \leq \lambda \leq \lambda_{0}
$$

with the normalization condition $\int d \lambda \rho(\lambda)=N$. One should note that near vicinity of the ground state, the level distribution functions may have large corrections, which may significantly change DOS near the ground state in comparison to the bulk distribution [14]. However, one can show that the main contribution to the probability (38) comes from the bulk of the distribution, where there is a large number of states and (43) is valid in the large $N$ limit. Combining (38) and (43), we obtain analogous to (39)

$$
\langle\Gamma(T)\rangle=\frac{\sigma^{2}}{\gamma} \sinh (\gamma T)\left\langle\frac{1}{\omega_{k 0}(0)}\right\rangle \frac{2 N}{\pi \lambda_{0}^{2}} \int_{0}^{2 \lambda_{0}} \frac{d \omega}{\omega} \sqrt{\omega\left(2 \lambda_{0}-\omega\right)} .
$$

From (44), it follows that the integral over the spectrum in the r.h.s. of (44) is accumulated at the upper bound of the integration, since the integrand only 
has integrable singularity at $\omega=0$. This indicates that the main contribution to (44) and therefore to (38), comes from the upper part of the spectrum. After evaluation of the integral, (44) yields

$$
\langle\Gamma(T)\rangle=\sinh (\gamma T)\left\langle\frac{1}{\omega_{k 0}(0)}\right\rangle \frac{2 N \sigma^{2}}{\gamma \lambda_{0}},
$$

which reduces to (39) after taking (41) into account. Based on the above arguments, we can employ the semicircle distribution to evaluate the average spacing $\left\langle\frac{1}{\omega_{k 0}(0)}\right\rangle$. This is done in Appendix $D$, and the probability (39) takes the form

$$
\langle\Gamma(T)\rangle=\frac{2}{\pi n} \lambda_{0}(T) \sinh (\gamma / T) .
$$

Since $\gamma T \sim 1$ and the absolute value of the ground state energy $\lambda_{0}(T)$ does not depend on $n$, it follows from (46) that the failure probability of the QAA is $\langle\Gamma(T)\rangle \sim 1 / n$, implying that the algorithm can be successful in the largen limit. As we will show in the subsequent section, this is a consequence of the Markovian nature of the Brownian motion model considered above. In the next section, we will discuss this in more details and compare to the results in the non-Markovian case.

The result (46) could not be obtained using a standard estimate (4) with $\Delta=\omega_{10}$ being the smallest gap between the ground state and the first excited level of the total Hamiltonian (?) As it follows from comparison of (38) and (34), the main contribution to the failure probability comes from the bulk of the spectrum, implying that the failure of the QAA occurs due to the interaction of the ground state with the "cloud" formed by all the excited states with $k=1, \ldots N$. In other words, it is the large number of possible transitions from the ground state that may cause the failure of the QAA, not the structure of the minimum gap in the energy spectrum. The main reason for this is that the phase factors present in (11) vanish after the integration over the angular variables (24). Note that these phase factors contain the transition frequencies and lead to the low transition probabilities in adiabatic regime. This is not the case in the Brownian motion model considered above, when all the excited levels work together and the excitation can be effectively viewed as a transition to the "cloud" of the excited states. This is in qualitative agreement with [13], confirming the conclusion that in the driven RMT models, the Landau-Zener mechanism of dissipation [12] is 
not important. Indeed, the theory of dissipation based on the Landau-Zener mechanism assumes [11] that the transitions happen due to the pairwise "level crossing" events, rather than the "collective" interactions picture that follows from our analysis.

In the regime when the QAA is successful considered above, the average minimal gap is $\Delta \simeq 1 / N$, and the formal application of the standard estimate gives $T \gtrsim N^{2}$, which is has nothing to do with the result (46). One should note that in some cases, the bound (4) can be improved by optimally adjusting the control parameter corresponding to the rate of the quantum adiabatic evolution [1]. In our case, this would correspond to introducing the time dependent rate parameter $\gamma(t)$ in (32), and then optimizing with respect to the function $\gamma(t)$. However, as we show in Appendix $E$, the result (38) is essentially invariant with respect to the choice of $\gamma(t)$, and therefore (46) does not depend on the functional form of the rate parameter. The main reason is that, according to the above discussion, the QAA fails due to the collective interaction of the ground adiabatic state with the cloud of excited states, and therefore there no well defined "dangerous" moments of time corresponding to the avoiding crossing [12], [11].

One may attempt to correct the standard estimate (4) using the average level spacing $\Delta=\left\langle\omega_{10}\right\rangle$ in the vicinity of the ground state instead of the average spacing. The point is that the spacing near vicinity of the edges of the spectrum may be significantly enhanced in comparison to the average one [14]. According to the above discussion, these estimates can not be correct, since they assume that the main contribution to the failure probability comes from the low-energy part of the spectrum.

\section{Motivations for Brownian Motion Model}

In this section, we will show that the specification considered in a previous section is actually quite general, since it can be mapped onto (2), with the appropriate choice of the "trajectory" parametrized by the functions $\alpha(t)$ and $\beta(t)$, and the problem Hamiltonian $H_{P}$ described by a random matrix belonging to the Gaussian Unitary ensemble (GUE). In general, such mapping yields the time-dependent coefficients $\{\gamma, \sigma\}$ characterizing the Ornstein-Uhlenbeck process. We will specify a particular parametrization that leads the constant coefficients, and therefore the stationary distributions.

Consider the QAEA (2) with a random GUE problem Hamiltonian $H_{P}=$ 
$V=H(T)$ and the driver $H_{D}=H(0)$. Since $V$ belongs to the GUE, its matrix elements are independent and Normally distributed, $V_{k j} \sim N\left(0, \sigma_{0}\right)$. Taking this and (2) into account, we derive the probability distribution for the matrix elements of the Hamiltonian $H(t)$ in the form

$$
P[H, t]=C_{N}(t) \prod_{k, j} \exp \left\{-\frac{\left[H_{k j}-\alpha(t) H(0)_{k j}\right]^{2}}{2 \sigma_{0}^{2} \beta^{2}(t)}\right\}
$$

where $C_{N}(t)$ is the normalization constant. Suppose there is a change of parametrization $\tau=\tau(t)$ in (2) that maps the total Hamiltonian dynamics (2) onto the Ornstein-Uhlenbeck process (12). The process (12) leads to the following probability distribution

$$
P[H, \tau]=[4 \pi D(\tau)]^{N^{2} / 2} \prod_{k, j} \exp \left\{-\frac{\left[H_{k j}-\bar{H}_{k j}(\tau)\right]^{2}}{4 D(\tau)}\right\},
$$

with

$$
\begin{aligned}
\bar{H}_{k j}(\tau) & =H(0)_{k j} \exp (-\gamma \tau), \\
D(\tau) & =\frac{1}{2} \int_{0}^{\tau} d \tau^{\prime} \sigma^{2}\left(\tau^{\prime}\right) \exp \left[-2 \gamma\left(\tau-\tau^{\prime}\right)\right]
\end{aligned}
$$

and we assumed that the effective variance $\sigma^{2}(\tau)$ may be time-dependent. Comparing (47) and (48), we obtain the conditions on the parameters $\gamma$ and $\sigma^{2}(\tau)$, in the form

$$
\begin{aligned}
\exp (-\gamma \tau) & =\alpha(t) \\
\int_{0}^{\tau} d \tau^{\prime} \sigma^{2}\left(\tau^{\prime}\right) \exp \left(2 \gamma \tau^{\prime}\right) & =\sigma_{0}^{2} \beta^{2}(t) \exp (2 \gamma t),
\end{aligned}
$$

and therefore 


$$
\begin{aligned}
\tau(t) & =-\frac{1}{\gamma} \ln [\alpha(t)] \\
\sigma^{2}(\tau) & =\sigma_{0}^{2} \exp (-2 \gamma \tau) \frac{d}{d \tau}\left\{\beta^{2}[t(\tau)] \exp (2 \gamma \tau)\right\}
\end{aligned}
$$

implying that the effective variance $\sigma^{2}(\tau)$ is indeed in general time-dependent. In what follows, we consider the QAEA (2)

$$
H(t)=H(0) \exp (-\gamma t)+V[1-\exp (-\gamma t)],
$$

where $y$ is a real parameter characterizing the speed of the parametric evolution. The parametrization (52) is a particular case of (2) with

$$
\alpha(t)=\exp (-\gamma t), \quad \beta(t)=1-\exp (-\gamma t), \quad T \sim \frac{1}{\gamma},
$$

and the parameters $\alpha(t)$ and $\beta(t)$ being the monotonic functions for $t \in$ $[0,+\infty]$ of (52). From (51), we derive the parameters of the corresponding Ornstein-Uhlenbeck process in the form

$$
\begin{aligned}
\tau(t) & =t \\
\sigma^{2}(t) & =2 \gamma \sigma_{0}^{2}[1-\exp (-\gamma t)],
\end{aligned}
$$

implying that the trajectories (52) are mapped onto the Ornstein-Uhlenbeck process with time-dependent variance parameter. From (54), it follows that the time dependence of the effective variance is slow and achieves the "saturation" during the runtime of the algorithm, when $t \sim 1 / \gamma$. As we show in Appendix $F$, there is a particular class of trajectories corresponding to the Ornstein-Uhlenbeck process with constant parameters [17].

As it was pointed out in [17], the mapping (51) is exact in a sense that the level distribution obtained from the corresponding Ornstein-Uhlenbeck model is identical to the level distribution of the parametrically driven Hamiltonian (2), at each instant of time. However, the actual level dynamics of the two models are quite different. For this reason, the level correlations calculated at different time moments are not equivalent for (12) and the corresponding 
(52). In particular, the driven system (52) contains the "quenched" disorder terms leading to the intertemporal correlations, which are absent in a Markovian system (12), [17]. In case of the QAEA with the random Problem Hamiltonian (52), the intertemporal correlators can be easily estimated. Making use of this and the knowledge of the effective level dynamics obtained by mapping onto (12), we will be able to estimate the probability of success for the model (52).

The probability of failure for the QAA is obtained from (11) in the form

$$
\Gamma(t)=\sum_{k=1}^{N} \int_{0}^{t} d t^{\prime} \int_{0}^{t} d t^{\prime \prime} \frac{\left[\dot{H}\left(t^{\prime}\right)\right]_{k 0}\left[\dot{H}\left(t^{\prime \prime}\right)\right]_{0 k}}{\omega_{k 0}\left(t^{\prime}\right) \omega_{k 0}\left(t^{\prime \prime}\right)} \exp \left\{-i \int_{t^{\prime}}^{t^{\prime \prime}} d \tau \omega_{k 0}(\tau)\right\}
$$

where we took into account the identity

$$
\left(\frac{\partial}{\partial t}\right)_{k 0}=\left\langle\phi_{k}(t)\left|\frac{\dot{H}(t)}{\omega_{k 0}(t)}\right| \phi_{0}(t)\right\rangle
$$

with $\dot{H}(t)=\frac{d H}{d t}$. In case (52), we have $\frac{d H}{d t}=-\gamma(H-V)$, and (5.5) yields

$$
\begin{aligned}
\Gamma(t) & =\gamma^{2} \sigma_{0}^{2} \sum_{k=1}^{N}\left|C_{k}(t)\right|^{2} \\
C_{k}(t) & =\int_{0}^{t} d t^{\prime} \frac{\exp \left\{-i \int_{0}^{t^{\prime}} d \tau^{\prime} \omega_{k 0}(\tau)\right\}}{\omega_{k 0}\left(t^{\prime}\right)}
\end{aligned}
$$

where we took into account the correlations $\left\langle V_{k 0}, V_{0 k}\right\rangle=\sigma_{0}^{2}$ and that the Hamiltonian $H(t)$ is diagonal in the adiabatic basis.

The second of (57) represents the transition amplitude, which typically contains two components. In the adiabatic limit, the leading contribution to the integral (57) comes from the endpoints of the integration and corresponds to the corrections to the adiabatic eigenstates due to the finite rate of the parametric transformation (52). The second contribution comes from the actual transitions between the "true" dynamic states with the non-adiabatic corrections to the states taken into account. The "transition" term is exponentially small in the adiabatic limit and exhibits the non-analytical dependence on the adiabaticity parameter ([6]). On the other hand, the "state 
correction" term typically has a power-law dependence on the adiabaticity parameter.

The general setting of the QAEA requires that the solution of the initial optimization problem is encoded in the adiabatic ground state of the Hamiltonian [1]. Therefore, in order to evaluate the success of the QAEA, one has to take into account both "correction" and "transition" contributions to the integral (57). The correction term gives the leading contribution and therefore determines the success of the QAEA. This distinguishes between the approach adopted in the present paper and the one from ([10]). In ([10]), the authors evaluated the failure of QAA based on the results ([11]) for the dissipation rate in the driven RMT ensembles. The dissipation was estimated in ([11]) based on the Landau-Zener theory, which takes only transition components in (57) into account. While this was a reasonable approximation to the estimation of the total dissipation rate, this approach is not applicable to the analysis of the QAA. The point is that the evaluation of the QAA performance requires taking into account any deviations from the adiabatic ground state, regardless whether they occur due to the "true" transitions to the "corrected" states, or the corrections to the states themselves. Therefore, it is essential that both contributions to (57) be taken into account. Moreover, from the above discussion, it follows that the "correction" terms give the leading contribution, and therefore the "transition" effects can be neglected in the adiabatic limit. This is consistent with the results of ([13]). The "correction" contribution in (57) is given by ([6])

$$
C_{k}(t)=\frac{-i}{\left[\omega_{k 0}(t)\right]^{2}}\left\{I-\exp \left[-i \int_{0}^{t} d t^{\prime} \omega_{k 0}\left(t^{\prime}\right)\right]\right\} .
$$

Combining (57) and (58), we obtain the failure probability of QAA

$$
\Gamma(T)=4 \gamma^{2} \sigma_{0}^{2} \sum_{k=1}^{N} \frac{1}{\left[\omega_{k 0}(T)\right]^{4}} \sin ^{2}\left[\frac{1}{2} \int_{0}^{T} d t^{\prime} \omega_{k 0}\left(t^{\prime}\right)\right] .
$$

In order to evaluate the failure probability (59), we have to specify the level dynamics of the driven model (52). Making use of the mapping (54) enables us to apply the optimal trajectory methods developed for the Brownian motion model. The optimal trajectories characterized by (36), and we obtain for the transition frequencies along the optimal trajectories in the form 


$$
\omega_{k 0}(t)=\omega_{k 0}(0) \frac{\sinh [\gamma(T-t)]}{\sinh (\gamma T)}+\omega_{k 0}(T) \frac{\sinh (\gamma t)}{\sinh (\gamma T)} .
$$

Substituting (60) into (59), we obtain

$$
\Gamma(T)=4 \gamma^{2} \sigma_{0}^{2} \sum_{k=1}^{N} \frac{1}{\left[\omega_{k 0}(T)\right]^{4}} \sin ^{2}\left[\frac{1}{2} \Phi_{k 0}(T)\right],
$$

with the total phase $\Phi_{k 0}(T)$ defined by

$$
\Phi_{k 0}(T)=\frac{2}{\gamma}\left[\frac{\omega_{k 0}(0)+\omega_{k 0}(T)}{2}\right] \operatorname{coth}\left(\frac{\gamma T}{2}\right) .
$$

Note that the total phase contains the large factor $1 / \gamma \sim T$, as expected. According to the estimates from the previous section, the minimal level separations are estimated as $\omega_{k 0}(0) \sim 1$ and $\omega_{k 0}(T) \sim \frac{1}{N}$. Since in adiabatic limit the rate of parametric evolution is much smaller than the initial transition frequency $\gamma<<\omega_{k 0}(0)$, the total phase is large and $\sin ^{2}\left[\frac{1}{2} \Phi_{k 0}(T)\right] \approx \frac{1}{2}$. Therefore, (61) reduces to

$$
\Gamma(T)=2 \gamma^{2} \sigma_{0}^{2} \sum_{k=1}^{N} \frac{1}{\left[\omega_{k 0}(T)\right]^{4}} .
$$

From (63), it follows that in order to evaluate the total failure probability of QAA, we need to know the density of states at the end of the evolution process. This is the consequence of the fact that the level separation is minimal at the end of the evolution. The final configuration of the optimal trajectory is given by (34) and corresponds to the standard GUE and the average density of states is given by the semicircle law (43). However, the expression (63) can not be evaluated in a continuous limit analogous to (38), since the corresponding integral diverges on the lower limit. This implies that the sum is accumulated on the lower limit corresponding to the lowfrequency transitions, and the structure of DOS near the lower edge of the spectrum may be important. As it was shown in [14], the DOS in GUE near the ground state has the form of the power law

$$
p(\lambda) \sim \lambda^{q(\lambda)}
$$


where the exponent $q(\lambda)=1 / 6$ in the narrow range near the ground state and tends to the limit $q(\lambda)=1 / 2$ corresponding to (43), outside that range. Making use of (64), the probability (63) can be estimated as

$$
\Gamma(T)=2 \gamma^{2} \sigma_{0}^{2} \sum_{k=1}^{N} \frac{1}{k^{4}\left[\Delta \omega_{k}\right]^{4}},
$$

with

$$
\Delta \omega_{k}=(1 / N)[\rho(\omega)]^{-1} \sim(1 / N)\left[\Delta \omega_{k}\right]^{-q} k^{-q} .
$$

Combining (65) and (66), we obtain

$$
\Gamma(T)=2 \gamma^{2} \sigma_{0}^{2} N^{p} \sum_{k=1}^{N} \frac{1}{k^{p}} \approx \frac{2}{p-1} \gamma^{2} \sigma_{0}^{2} N^{p},
$$

where $p=4 /(1+q)>2$. The estimate of the power of QAA is defined from the requirement that the failure probability (67) is small. Taking into account (53) and that in the GUE $\sigma_{0}^{2} \sim 1 / N$, we estimate the runtime of the QAA

$$
T>N^{a}, \quad a=\frac{3-q}{2(1+q)},
$$

where the exponent $q$ comes from (64). From (68), it follows that $17 / 14 \leq$ $a \leq 5 / 6$, where the upper and lower limits correspond to $q=1 / 6$ and $q=1 / 2$, respectively. The estimate (68) implies the exponential complexity of the QAA with $a \simeq 1$.

Comparing (46) and (67), we observe that the Markovian evolution model (12) may lead to polynomial complexity, whereas the driven RMT model (52) always leads to the exponential complexity of QAEA. This has a simple intuition. According to the above discussion, the main difference between the models (12) and (52) comes from different intertemporal level correlations. In the Markovian model (12), there are only short-range correlations, whereas in the driven RMT model (52), the long-range intertemporal correlations are present. On one hand, this leads to the complete cancellation of phases, which removes the exponentially small factors for the transitions to the highly excited states and leads to the diffusion type of dissipation process in (12). On the other hand, the baseness of the long-range intertemporal correlations leads to much weaker effective interaction between the states in the 
Markovian model (12) as opposed to (52). The results (??) and (68) indicate that this "weakness" of effective transitions is the leading effect that may in principle make the Markovian type QAEA (12) successful.

\section{Conclusion}

We analyze the power of quantum adiabatic evolution algorithms (QAEA) for solving random NP-hard optimization problems within a theoretical framework based on the random matrix theory (RMT). We present two types of the driven RMT models.

In the first model, the "driving" Hamiltonian is represented by Brownian motion in the matrix space. We use the Brownian motion model to obtain a description of multiple avoided crossing phenomena. The model allows for the close-form analytical treatment in adiabatic approximation, within the method of optimal trajectories [23]. We show that the failure mechanism of the QAA is due to the interaction of the ground state with the "cloud" formed by all the excited states. This confirms that in the driven RMT models, the Landau-Zener mechanism of dissipation based on the assumption of pairwise level interactions, is not important ([13]). We show that the QAEA may have a finite probability of success in a certain range of parameters, implying the possibility of polynomial complexity of the algorithm. This model can be viewed as a relatively "mild" test on the general QAA performance for the computationalily hard optimization problems. In this case, the quantum evolution is not affected by the "accidental" level crossing phenomenon that may lead to the QAA failure in standard models [11], and the only reason for the possible QAA failure is a complex structure of the problem Hamiltonian representing the cost function.

The second model corresponds to the standard QAEA with the problem Hamiltonian taken from the Gaussian Unitary RMT ensemble (GUE). We show that the eigenvalues and eigenvector dynamics in this model can be mapped onto the dynamics in the Brownian motion model considered before. This enables us to apply the optimal trajectories approach developed for the previous case, to the driven RMT model. However, the driven RMT

5 and the Markovian Brownian motion models have different structure of the intertemporal level correlations, and this leads to different performance of the QAEA. The Brownian motion model may lead to polynomial complexity, whereas the driven RMT model always gives the exponential complexity

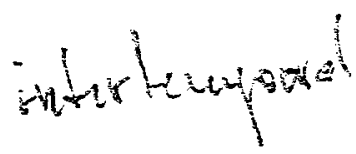


of the algorithm. In the Markovian Brownian motion model, there are only short-range correlations, whereas in the driven RMT model, the long-range intertemporal correlations are present. The absence of the long-range intertemporal correlations leads to much weaker effective interaction between the states in the Markovian model (12) as opposed to (52). Our results indicate that this "weakness" of effective transitions is the leading effect that may in principle make the Markovian type QAEA (12) successful. 


\section{Appendix A}

According to (30), the effective potential is given by

$$
U_{e f f}(\Lambda)=-\frac{1}{2} \sum_{k}\left(v_{k}^{2}(\Lambda)+\sigma^{2} \frac{\partial v_{k}(\Lambda)}{\partial \lambda_{k}}\right),
$$

with the velocities $v_{k}(\Lambda)$ defined by (22). From (22) and (69), we obtain

$$
\begin{aligned}
U_{e f f}(\Lambda)= & -\frac{\gamma^{2}}{2} \sum_{k} \lambda_{k}^{2}-\gamma \lambda_{k} \sum_{k, l \neq k} \frac{\sigma^{2}}{\lambda_{k}-\lambda_{l}}- \\
& -\frac{1}{2}\left[\sum_{k, l \neq k, l^{\prime} \neq k} \frac{\sigma^{4}}{\left(\lambda_{k}-\lambda_{l}\right)\left(\lambda_{k}-\lambda_{l^{\prime}}\right)}-\sum_{k, l \neq k} \frac{\sigma^{4}}{\left(\lambda_{k}-\lambda_{l}\right)^{2}}\right] .
\end{aligned}
$$

Under the cyclic permutation of the indices $\left\{k, l . l^{\prime}\right\}$, we have

$$
\frac{1}{\left(\lambda_{k}-\lambda_{l}\right)\left(\lambda_{k}-\lambda_{l^{\prime}}\right)}+\frac{1}{\left(\lambda_{l}-\lambda_{l^{\prime}}\right)\left(\lambda_{l}-\lambda_{k}\right)}+\frac{1}{\left(\lambda_{l^{\prime}}-\lambda_{k}\right)\left(\lambda_{l^{\prime}}-\lambda_{l}\right)}=0
$$

therefore the first term in the square brackets in the r.h.s. of (70) reduces to

$$
\sum_{k, l \neq k, l^{\prime} \neq k} \frac{\sigma^{i}}{\left(\lambda_{k}-\lambda_{l}\right)\left(\lambda_{k}-\lambda_{l^{\prime}}\right)}=\sum_{k, l \neq k} \frac{\sigma^{4}}{\left(\lambda_{k}-\lambda_{l}\right)^{2}},
$$

and the two terms in the square brackets in (70) sum up to zero. The second term in the r.h.s. of (70) is given by

$$
\gamma \sigma^{2} \sum_{k, l \frac{1}{\tau} k} \frac{\lambda_{k}}{\lambda_{k}-\lambda_{l}}=\gamma \sigma^{2} \frac{N(N-1)}{2}=\text { const. }
$$

Combining (70), (71) and (72) finally yields

$$
U_{e f f}(\Lambda)=-\frac{\gamma^{2}}{2} \sum_{k} \lambda_{k}^{2}
$$

identical to (31) from the text. 


\section{Appendix $\mathrm{B}$}

According to (32), the optimal trajectories satisfy

$$
\ddot{\lambda_{k}}(t)-\gamma^{2} \lambda_{k}=0
$$

The equations (32) are of the second order and therefore require two initial (or boundary) conditions for each $k=0, \ldots N$. Suppose that we only have one initial condition for the optimal trajectories

$$
\lambda_{k}(t=0)=\lambda_{k}(0)
$$

for each $k$ and the final points $\lambda_{k}(t=T)$ are not fixed. Following the standard procedure [9], we impose the additional "transversality" boundary conditions at the final points

$$
\left(\frac{\partial L}{\partial \dot{q}_{i}}\right)_{t=T}=0
$$

which yield the boundary conditions for our problem

$$
\dot{\lambda_{k}}(T)=v_{k}[\Lambda(T)]=-\gamma \lambda_{k}(T)+\left(\sum_{l \neq k} \frac{\sigma^{2}}{\lambda_{k}(T)-\lambda_{l}(T)}\right)
$$

The equations (32) with (74) and (76), provide a close set. From (32) with (74) and (76), we obtain

$$
\lambda_{k}(t)=\lambda_{k}(0) \frac{\sinh [\gamma(T-t)]}{\sinh (\gamma T)}+\lambda_{k}(T) \frac{\sinh (\gamma t)}{\sinh (\gamma T)} .
$$

The final "coordinates" $\left\{\lambda_{k}(T)\right\}$ are defined from (32) and (76) as solutions of the self-consistent equation

$$
\lambda_{k}(T)=\lambda_{k}(0) \exp (-\gamma T)+\frac{[1-\exp (-2 \gamma T)]}{\gamma}\left(\sum_{j \neq k} \frac{\sigma^{2}}{\lambda_{k}(T)-\lambda_{j}(T)}\right)
$$

which indicates that in the large-time limit $\gamma T>>1$, the eigenvalues $\left\{\lambda_{k}(T)\right\}$ are separated, i.e. the degeneracy is completely lifted, even though the initial distribution $\left\{\lambda_{k}(0)\right\}$ may have a significant degree of degeneracy. Specifically, (78) leads to the standard equilibrium GUE distribution described by (34) in the large-time limit. 


\section{Appendix $\mathrm{C}$}

In this Appendix, we will derive the equilibrium $N$-particle distribution for the levels and show that it is indeed an equilibrium GUE distribution for any choice of the "rate" parameter $\alpha(t)$ introduced in the text. According to the first of equation (18), the non-stationary $N$-level distribution function $P(\Lambda, t)$ satisfies the Fokker-Plank equation [18]

$$
\frac{\partial P(\Lambda, t)}{\partial t}+\frac{\partial}{\partial \lambda_{k}}\left[v_{k}(\Lambda) P(\Lambda, t)-\frac{\sigma^{2}}{2} \frac{\partial P(\Lambda, t)}{\partial \lambda_{k}}\right]
$$

and the equilibrium distribution is given by

$$
P_{e q}(\Lambda)=C \exp \left[-\frac{2}{\sigma^{2}} V_{e f f}(\Lambda)\right]
$$

with a normalization constant $C$ and the effective potential

$$
V_{e q}(\Lambda)=-\int^{\Lambda} d \lambda_{k} v_{k}(\Lambda)=\frac{\gamma}{2} \sum_{k} \lambda_{k}^{2}-\sigma^{2} \sum_{k, l \neq k} \log \left|\lambda_{k}-\lambda_{l}\right| .
$$

Combining (80) and (81), we finally obtain

$$
P_{e q}(\Lambda)=C \exp \left[\sum_{l, l \neq l, k} \log \left(\lambda_{k}-\lambda_{l}\right)^{2}-\frac{\gamma}{\sigma^{2}} \sum_{k} \lambda_{k}^{2}\right],
$$

corresponding to the standard GUE distribution [7] for any choice of parameters $\gamma$ and $\sigma$.

\section{$9 \quad$ Appendix D}

In this Appendix, we employ the semicircle distribution to evaluate the probability (38). According to (5), the levels of $H(0)=H_{D}$ are equally spaced with $\omega_{k 0}(0)=k$ and the degeneracy of the $k$-th level is

$$
g_{k}=\left(\frac{n}{k}\right)=\frac{n !}{(n-k) ! k !} .
$$

In the continuous limit of (43), we have 


$$
\omega_{k 0}(0)=k(\omega)=\frac{n}{\lambda_{0}} \omega
$$

where the last equality follows from the observation that the width of the spectrum at $t=0$ is $2 \omega_{n 0}(0)=2 n$ and there are $2 n+1$ distinct levels, whereas the spectrum at $t=T$ is bounded within the range of $2 \lambda_{0}$. Combining (38) and (43), we obtain

$$
\langle\Gamma(T)\rangle=\frac{\sigma^{2}}{\gamma} \sinh (\gamma T) \frac{2 N}{\pi \lambda_{0}^{2}} \int_{0}^{2 \lambda_{0}} \frac{d \omega}{\omega} \sqrt{\omega\left(2 \lambda_{0}-\omega\right)} \frac{g_{k(\omega)}}{k(\omega)},
$$

with the degeneracy $g_{k(\omega)}$ given by (83) and (84). In the large $n$ limit, the degeneracy (83) has a sharp peak at $k=n / 2$, and (85) reduces to

$$
\langle\Gamma(T)\rangle=\frac{\sigma^{2}}{\gamma} \sinh (\gamma T) \frac{2 N}{\pi \lambda_{0}^{2}} \int_{0}^{2 \lambda_{0}} \frac{d \omega}{\omega} \sqrt{\omega\left(2 \lambda_{0}-\omega\right)} \frac{\lambda_{0}}{n} \frac{g(\omega)}{\omega},
$$

with

$$
g(\omega)=\sqrt{\frac{n}{2 \pi}} \exp \left[-\frac{n\left(\omega-\lambda_{0}\right)^{2}}{2 \lambda_{0}^{2}}\right]
$$

Evaluating (86) in the large $n$ limit, we obtain

$$
\langle\Gamma(T)\rangle=\frac{2}{\pi n} \frac{\sigma^{2}}{\gamma} \sinh (\gamma T) \frac{2 N}{\lambda_{0}},
$$

which reduces to (46) after taking (41) into account.

\section{Appendix E}

In this Appendix, we show that the estimate (??) for the power of the QAA algorithm given in the text for a particular case of the constant parameter characterizing the rate of changing the Hamiltonian $\alpha \equiv$ const, can not be improved by introducing the time-dependent rate $\chi(t)$.

The failure probability of the QAA is given by 


$$
\langle\Gamma(t)\rangle=\sum_{k=1}^{N} \int_{0}^{t} d t^{\prime} \frac{\sigma^{2}}{\left[\omega_{k 0}\left(t^{\prime}\right)\right]^{2}},
$$

where $\omega_{k 0}(t)=\lambda_{k}(t)-\lambda_{0}(t)$ are the transition frequencies defined corresponding to the optimal trajectories $\left\{\lambda_{k}(t)\right\}$. Under the transformation (??) with time-dependent evolution rate $\gamma(t)$, the optimal trajectories satisfy, analogous to (32)

$$
\ddot{\lambda_{k}}(t)-\gamma^{2}(t) \lambda_{k}=0 .
$$

Analogous to (36), the solution of (90) can be presented in the form

$$
\lambda_{k}(t)=\lambda_{k}(0) \varphi_{1}(t)+\lambda_{k}(T) \varphi_{2}(t),
$$

where $\left\{\varphi_{1}(t), \varphi_{2}(t)\right\}$ are the two linearly-independent solutions of the secondorder ODE (90) satisfying the following boundary conditions

$$
\begin{array}{ll}
\varphi_{1}(0)=1 ; & \varphi_{1}(T)=1 ; \\
\varphi_{2}(0)=0 ; & \varphi_{2}(T)=1 .
\end{array}
$$

In this case, it is well known [24] that for the system (90), the Wronskian $W(-i)$ defined by

$$
W(\gamma)=\varphi_{1}(t) \frac{d \varphi_{2}(t)}{d t}-\varphi_{2}(t) \frac{d \varphi_{1}(t)}{d t}=\left[\varphi_{2}(t)\right]^{2} \frac{d}{d t}\left[\frac{\varphi_{1}(t)}{\varphi_{2}(t)}\right]
$$

is an integral of motion. Substituting (91) into (89), we obtain analogous to (37)

$$
\langle\Gamma(T)\rangle=\sum_{k=1}^{N} \int_{0}^{T} d t \frac{\sigma^{2}}{\left[\omega_{k 0}(0) \varphi_{1}(t)+\omega_{k 0}(T) \varphi_{2}(t)\right]^{2}} .
$$

Making use of (93), (94) reduces to

$$
\langle\Gamma(T)\rangle=\sum_{k=1}^{N} \int_{0}^{+\infty} \frac{d u}{W(\gamma)} \frac{\sigma^{2}}{\left[\omega_{k 0}(0) u+\omega_{k 0}(T)\right]^{2}},
$$


where we made the substitution $u=\varphi_{1}(t) / \varphi_{2}(t)$ and took (92) into account. After the integration (95) yields

$$
\langle\Gamma(T)\rangle=\frac{\sigma^{2}}{W(\gamma)} \sum_{k=1}^{N} \frac{1}{\omega_{k 0}(0) \omega_{k 0}(T)},
$$

leading to the same bound on the runtime as the estimate (??). Note that for the case of constant $\chi=1 / T$ considered in the text, the basis functions $\left\{\varphi_{1}(t), \varphi_{2}(t)\right\}$ lead to $(36)$, and the Wronskian is given by $W(\gamma)=\gamma / \sinh (\gamma)$. In this case, (96) reduces to (38) from the text.

\section{Appendix F}

Following [17], consider the QAEA (2)

$$
H(t)=\cos (\Omega t) H_{0}+\sin (\Omega t) V,
$$

where $\Omega$ is a real parameter characterizing the "speed" of the parametric evolution. The parametrization (97) is a particular case of (2) with

$$
\alpha(t)=\cos (\Omega t), \quad \beta(t)=\sin (\Omega t), \quad T=\frac{\pi}{2 \Omega},
$$

and the parameters $\alpha(t)$ and $\beta(t)$ being the monotonic functions of time during the runtime $t \in[0, T]$ of (97). From (51), we derive the parameters of the corresponding Ornstein-Uhlenbeck process in the form

$$
\begin{aligned}
\tau(t) & =-\frac{1}{\gamma} \ln [\cos (\Omega t)] \\
\sigma^{2} & =2 \gamma \sigma_{0}^{2}
\end{aligned}
$$

implying that the trajectories (97) are mapped onto the Ornstein-Uhlenbeck process with constant parameters [17]. 


\section{References}

[1] E. Farhi, J. Goldstone, S. Gutmann, and M. Sipser, "Quantum Computation by Adiabatic Evolution", arXiv:quant-ph/0001106, (2002).

[2] E. Farhi, J. Goldstone, S. Gutmann, "Quantum Adiabatic Evolution Algorithms versus Simulated Annealing", arXiv:quant-ph/0201031 v1, (2002).

[3] E. Farhi, J. Goldstone, S. Gutmann, and M. Sipser, "Quantum Adiabatic Evolution Algorithms with Different Paths", arXiv:quantph/0208135, (2002).

[4] E. Farhi, J. Goldstone, S. Gutmann, "Quantum adiabatic evolution algorithms with different paths", arXiv:quant-ph/0208135.

[5] A. Boulatov and V. N. Smelyanskiy, "Quantum Adiabatic Algorithms and Large Spin Tunneling", Phys. Rev. A 68, 062321 (2003).

[6] L.D. Landau and E.M. Lifschitz, Quantum Mechanics (Pergammon, Oxford, 1976).

[7] F. J. Dyson, J. Math. Phys. (N.Y.) 3, 140 (1962).

[8] F. J. Dyson, J. Math. Phys. (N.Y.) 3, 166 (1962).

[9] L.D. Landau and E.M. Lifschitz, Mechanics (Pergammon, Oxford, 1972).

[10] D. R. Mitchell, C. Adami, and C. P. Williams, "A Random Matrix Analysis of Adiabatic Quantum Computing", (unpublished).

[11] M. Wilkinson, "Statistical aspects of dissipation by Landau-Zener transitions", J. Phys. A: Math. Gen. 21, 4021 (1988).

[12] Zener, Proc. Roy. Soc. A 137, 606 (1932).

[13] A. Bulgac, G. D..Dang, and D. Kusnezov, "Random matrix approach to quantum dissipation", Phys. Rev. E., v.54, 3468 (1996).

[14] B. V. Bronk, "Accuracy of the Semicircle Approximation for the Density of Eigenvalues of Random Matrices", Journ. Math. Phys., 5, 215 (1964). 
[15] I. E. Smolyarenko and B. D. Simons, ."Parametric Spectral Statistics in Unitary Random Matrix Ensembles: From Distribution Functions to Intra-Level Correlations", arXiv:cond-mat/0303475 v1, (2003).

[16] I. E. Smolyarenko, F. M. Marchetti, and B. D. Simons, Phys. Rev. Lett. 88,256808 (2002).

[17] O. Narayan and B. S. Shastry, "Dyson's Brownian Motion and Universal Dynamics of Quantum Systems", Phys. Rev. Lett. 71, 2106 (1993).

[18] C. W. J. Beenakker and B. Rejaei, ."Random-matrix theory of parametric correlations in the spectra of disordered metals and chaotic billiards", arXiv:cond-mat/9310068, (1993).

[19] P. Pechukas, "Distribution of Energy Eigenvalues in the Irregular Spectrum", Phys. Rev. Lett. 51, 943 (1983).

[20] T. Yukawa, "New Approach to the Statistical Properties of Energy Levels", Phys. Rev. Lett. 54, 1883 (1985).

[21] R.P. Feynman, Statistical Mechanics (West View Press, New York, 1998).

[22] H. Kleinert, Path Integrals in Quantum Mechanics, Statistics, and Polymer Physics, (World Scientific, Singapore, 1995).

[23] R.P. Feynman, A.R. Hibbs, Quantum Mechanics and Path Integrals, (McGraw Hill, New York, 1965).

[24] V.I. Arnold, Ordinary Differential equations, (MIT Press, Cambridge, 1978).

[25] G. E. Santoro, R. Martonak, E. Tosatti, R. Car, "Theory of quantum annealing of an Ising Spin Glass", Science 295, p. 2447 (2002).

[26] J. Brooke, D. Bitko, T.F. Rosenbaum, G. Aepple, "Quantum annealing of a disordered magnet", Science 284, p. 779 (1999).

[27] M.R. Garey and D.S. Johnson, Computers and Intractability. A Guide to the Theory of NP-Completeness (W.H. Freeman, New York, 1997) 
[28] E. Farhi, J. Goldstone, S. Gutmann, J. Lapan, A. Lundgren, and D. Preda, Science 292, 472 (2001).

[29] E. Farhi, J. Goldstone, and S. Gutmann, arXiv:quant-ph/0007071.

[30] A. M. Childs, E. Farhi, J. Goldstone, and S. Gutmann, arXiviquant$\mathrm{ph} / 0012104$.

[31] T.Hogg, "Adiabatic Quantum Computing for Random Satisfiability Problems", arXiv:quant-ph/0206059.

[32] W. Van Dam, M. Mosca, U. Vazirani, "How Powerful is adiabatic Quantum Computation?", arXiv:quant-ph/0206003.

[33] C. Bennett, E. Bernstein, G. Brassard, and U. Vazirani,"Strengths and weaknesses of quantum computing", SIAM Journal of Computing, 26, pp. 1510-1523 (1997); arXiv:quant-ph/9701001.

[34] W. Van Dam, M. Mosca, U. Vazirani, "How Powerful is Adiabatic Quantum Computation?", FOCS 2001. 